\title{
Physician Distribution by Specialty and Practice Setting: Findings in Japan in 2000, 2010 and 2016
}

\author{
Ryo Ikesu, ${ }^{1}$ Atsushi Miyawaki ${ }^{1}$ and Yasuki Kobayashi ${ }^{1}$ \\ ${ }^{1}$ Department of Public Health, Graduate School of Medicine, The University of Tokyo, Tokyo, Japan
}

\begin{abstract}
As the medical demand is projected to increase along with the population aging in Japan, the geographical distribution of physicians is a significant concern for society and policymakers. To implement effective measures on geographical physician distribution, this study aimed to describe and compare the distribution of physicians by specialty in 2000, 2010 and 2016 in Japan, and examine whether practice setting was associated with distribution. To quantify the geographical physician distribution by specialty, we calculated the Gini coefficients of physicians working at clinics or hospitals in 2000, 2010, and 2016. We used the basic geographic unit for medical care planning in Japan, a secondary medical area, as the study unit. To show the association between the geographical distribution of physicians in each specialty and their practice setting, we categorized specialties into two groups by the proportion of physicians in that specialty working in hospitals, and showed aggregated Lorenz curves for each category. The overall geographical distribution of physicians appeared to improve during the study period, but varied by specialty. Those in specialties, where at least $90 \%$ of physicians work in hospitals such as anesthesiologists and radiologists, were more clustered, as shown by the Lorenz curves and the Gini coefficients. Similar distributional differences were also found even when we excluded physicians working in clinics, meaning that the distributional variation could be explained by other factors than the distribution of hospitals. These results suggest that the nature of practice in each specialty strongly affects the geographical distribution of specialists.
\end{abstract}

Keywords: delivery of health care; health care facilities, manpower, and services; Japan; medicine; physicians Tohoku J. Exp. Med., 2020 May, 251 (1), 1-8.

\section{Introduction}

The geographical distribution of physicians is a significant concern for society and policymakers (Newhouse 1990; Kobayashi and Takaki 1992; Hann and Gravelle 2004; Horev et al. 2004; Rosenthal et al. 2005; Guttmann et al. 2010; Isabel and Paula 2010; Goodfellow et al. 2016). Physicians play an essential role in maintaining people's health. Previous studies have suggested that physician supply can have positive effects on health outcomes such as life expectancy in many countries (Guttmann et al. 2010; Chang et al. 2011, 2017; Sakai et al. 2016). However, the geographical distribution of physicians does not necessarily match the geographical distribution of populations who need healthcare, especially in rural areas (MacDowell et al. 2010; Huang and Finegold 2013; Petterson et al. 2013; Sharma 2015). Rural areas still suffer from physician short- ages, which leads to decreased access to healthcare services (MacDowell et al. 2010; Sharma 2015).

The place where physicians practice also varies between specialties. For example, radiologists' work requires expensive medical equipment such as computed tomography, magnetic resonance imaging, and positron emission tomography scanners. These physicians therefore almost inevitably work in urban medical centers. This means that distribution of physicians may vary by specialty. Previous studies, however, have focused on overall physician distribution (Kobayashi and Takaki 1992) or specific specialties such as pediatrics (Chang and Halfon 1997), and have seldom examined the differences in physician distribution by the nature of the specialty. The total number of physicians is regulated in developed countries (Simoens and Hurst 2006), and thus understanding and comparing the characteristics of physician distribution by specialty is

Received February 26, 2020; revised and accepted April 15, 2020. Published online May 1, 2020; doi: 10.1620/tjem.251.1.

Correspondence: Ryo Ikesu, M.D., Department of Public Health, Graduate School of Medicine, The University of Tokyo, 7-3-1 Hongo,

Bunkyo-ku, Tokyo 113-0033, Japan.

e-mail: ikesu-tky@umin.ac.jp

(C)2020 Tohoku University Medical Press. This is an open-access article distributed under the terms of the Creative Commons Attribution-NonCommercial-NoDerivatives 4.0 International License (CC-BY-NC-ND 4.0). Anyone may download, reuse, copy, reprint, or distribute the article without modifications or adaptations for non-profit purposes if they cite the original authors and source properly.

https://creativecommons.org/licenses/by-nc-nd/4.0/ 
essential for policymakers to implement effective policies on geographical physician distribution.

In this study, we therefore aimed to describe and compare the physician distribution by specialty in 2000, 2010 and 2016 in Japan, and examine whether the practice setting was associated with physician distribution.

\section{Materials and Methods}

Data

The Physician, Dentist, and Pharmacist Census is a biennial survey, to which all physicians in Japan are required by law to submit their specialty and place of work. Under the permission of the Ministry of Health, Labour and Welfare (Seito-0913-5), we obtained anonymized individual data from this census. The numbers of physicians working in clinics or hospitals in 2000, 2010, and 2016 were extracted using the individual census data. The numbers of particular types of specialists were also extracted, including internists (allergy physicians, cardiologists, diabetologists, gastroenterologists, hematologists, infectious disease physicians, internal physicians, nephrologists, neurologists, psychotherapists, pulmonologists, and rheumatologists), surgeons (breast surgeons, cardiac surgeons, colon surgeons, cosmetic surgeons, gastric surgeons, plastic surgeons, respiratory surgeons, and tracheoesophageal surgeons), orthopedists, obstetricians and gynecologists (OB/GYNs), pediatricians, psychiatrists, brain surgeons, ophthalmologists, dermatologists, otolaryngologists, urologists, anesthesiologists, pathologists, radiologists, emergency physicians, and rehabilitation physicians. The numbers of internists, surgeons, pathologists, and emergency physicians were not available for 2000 , because the specialty categorization in the census for that year was not consistent with that used in 2010 and 2016. Consents from the physicians were waived because the data were used in an anonymized format. Population data for each municipality in 2000, 2010, and 2016 were obtained from the Basic Resident Registration Network System (Japan's compulsory official registration system) (Ministry of Internal Affairs and Communications 2017).

\section{Geographical unit for analysis}

We used the secondary medical area (SMA) as the geographical study unit. SMAs are geographic units used for medical care planning in Japan, and usually cover several municipalities. In each SMA, the total number of beds offering general inpatient care is regulated by the Medical Care Act, based on the population size and structure (age, morbidity, cross-boundary flows) (Ministry of Health, Labour and Welfare 2017). There were 344 SMAs at the end of 2016, and we used 342 of them for this study. We excluded two SMAs (accounting for approximately $0.5 \%$ of the total population in Japan) for which we were unable to obtain population data for the study period because of a split of municipalities. To make the study results comparable throughout the study period, we applied the boundaries of municipalities at the end of 2016 to all the data and then defined each SMA based on the boundaries of SMAs in 2016.

\section{Statistical analysis}

We used the Gini coefficient to represent the physician distribution. Originally, the Gini coefficient was used to quantify income inequality within a particular group (Dalton 1920). It ranges from 0 to 1 , and a larger coefficient shows a more uneven distribution of income. Previous studies have made use of this statistic to show physician distribution (Kobayashi and Takaki 1992; Hann and Gravelle 2004; Horev et al. 2004; Isabel and Paula 2010). We also used the Gini coefficient to describe the physician distribution for each specialty.

The Gini coefficients were calculated based on the number of physicians in the specialty by population size. For each of 2000, 2010, and 2016, we calculated the ratio of each type of specialist to the population in each SMA. We showed the 342 SMAs in ascending order of this ratio and used Lorenz curves to derive the Gini coefficients.

We divided the specialties into two groups based on post-hoc classification of dependence on hospital-based practice, which was determined by the proportion of physicians working exclusively in hospitals to those working in clinics or hospitals. For 2010 and 2016, a specialty was categorized as "hospital-dependent" if the proportion of physicians working in hospitals was at least 0.9 . We developed aggregated Lorenz curves for each category, to examine whether practice setting was associated with physician distribution. To rule out the possibility that the distribution of hospital-dependent physicians was solely determined by the distribution of hospitals, we calculated specialty-specific Gini coefficients for physicians working only in hospitals, excluding those working in clinics.

To examine whether each type of specialist was appropriately distributed throughout Japan, we calculated specialty-specific Gini coefficients using prefectures as the study unit. Japan has 47 prefectures, and they are larger geographic units than SMAs, which are composed of a group of municipalities in each prefecture (Ministry of Health, Labour and Welfare 2017). Prefectures are also used for medical care planning purposes, and known as tertiary medical areas, except for Hokkaido, which is the largest prefecture in Japan and has six tertiary medical areas. It is therefore likely that distributional differences between prefectures reflected unequal physician distribution because prefectures are required to deliver comprehensive medical care to their residents.

All analyses used R 3.4.1. This study was approved by the ethics committee at the Graduate School of Medicine and Faculty of Medicine, University of Tokyo (no. 2018069NI, approved on February 22, 2019). 


\section{Results}

\section{Study population}

Table 1 shows the numbers of physicians in the 342 SMAs in the three years of the study. Across the three years, the overall number of physicians increased. The number of physicians in each specialty also increased or remained stable. In particular, the numbers of anesthesiolo- gists, psychiatrists, radiologists, and rehabilitation physicians increased dramatically from 2000 to 2016.

Table 2 shows the trends in SMAs' population over the three years (for the 342 SMAs). The population of the smallest SMA was about 20,000, and that of the largest one was approximately $2,500,000$. The median and minimum population decreased, suggesting that the population of smaller SMAs probably decreased over the study period.

Table 1. Numbers of physicians in 2000, 2010 and 2016, by specialty ${ }^{1)}$.

\begin{tabular}{|c|c|c|c|c|}
\hline & $2000^{4)}$ & 2010 & 2016 & $\begin{array}{c}\text { Increase from } \\
2000 \text { to } 2016(\%)\end{array}$ \\
\hline All doctors & 252,546 & 293,474 & 317,808 & 25.8 \\
\hline Doctors working in clinics or hospitals & 240,174 & 278,923 & 303,148 & 26.2 \\
\hline Internists $^{2)}$ & NA & 104,541 & 113,163 & - \\
\hline Surgeons ${ }^{3)}$ & NA & 29,566 & 30,175 & - \\
\hline Orthopedists & 17,746 & 19,851 & 21,166 & 19.3 \\
\hline Obstetricians/gynecologists & 12,257 & 12,289 & 13,067 & 6.6 \\
\hline Pediatricians & 13,932 & 15,777 & 16,830 & 20.8 \\
\hline Psychiatrists & 10,936 & 14,127 & 15,530 & 42.0 \\
\hline Brain surgeons & 5,995 & 6,647 & 7,311 & 22.0 \\
\hline Ophthalmologists & 11,888 & 12,720 & 13,068 & 9.9 \\
\hline Dermatologists & 7,270 & 8,430 & 9,062 & 24.6 \\
\hline Otolaryngologists & 9,020 & 8,977 & 9,214 & 2.2 \\
\hline Urologists & 5,667 & 6,478 & 7,021 & 23.9 \\
\hline Anesthesiologists & 5,694 & 7,677 & 9,111 & 60.0 \\
\hline Pathologists & NA & 1,505 & 1,882 & - \\
\hline Radiologists & 4,453 & 5,562 & 6,549 & 47.1 \\
\hline Emergency physicians & NA & 2,254 & 3,226 & - \\
\hline Rehabilitation physicians & 1,261 & 1,903 & 2,479 & 96.6 \\
\hline Other clinicians & NA & 20,619 & 24,294 & - \\
\hline
\end{tabular}

NA, not available.

1) The number of physicians in each specialty was calculated as the sum of those in each SMA used in this study. We successfully extracted the physician data for almost all the SMAs (342 out of 344).

${ }^{2}$ Internists included allergy physicians, cardiologists, diabetologists, gastroenterologists, hematologists, infectious disease internists, general internal physicians, nephrologists, neurologists, psychotherapists, pulmonologists, and rheumatologists.

${ }^{3}$ Surgeons included breast surgeons, cardiac surgeons, colon surgeons, cosmetic surgeons, gastric surgeons, general surgeons, plastic surgeons, respiratory surgeons, and tracheoesophageal surgeons.

${ }^{4)}$ We were unable to extract data for internists, surgeons, pathologists, and emergency physicians in 2000 because of data availability.

Table 2. Summary statistics for the population of the secondary medical areas.

\begin{tabular}{lrrr}
\hline & \multicolumn{1}{c}{2000} & \multicolumn{1}{c}{2010} & \multicolumn{1}{c}{2016} \\
\hline Minimum & 25,228 & 21,936 & 20,422 \\
Median & 239,737 & 231,594 & 220,734 \\
Mean & 367,299 & 369,201 & 365,336 \\
Maximum & $2,474,579$ & $2,537,920$ & $2,565,982$ \\
Total population & $125,616,277$ & $126,266,695$ & $124,944,752$ \\
$\mathrm{n}$ & 342 & 342 & 342 \\
\hline
\end{tabular}

Total population was calculated as the sum of the population of each SMA used in this study. We successfully extracted population data for almost all the SMAs (342 out of 344). 
R. Ikesu et al.

Table 3. Gini coefficient and hospital-dependency of each specialty ${ }^{1)}$.

\begin{tabular}{|c|c|c|c|c|c|}
\hline & \multicolumn{3}{|c|}{ Gini coefficients } & \multicolumn{2}{|c|}{ Proportion of hospital physicians } \\
\hline & $2000^{4)}$ & 2010 & 2016 & 2010 & 2016 \\
\hline All doctors & 0.227 & 0.220 & 0.217 & - & - \\
\hline Doctors working in clinics or hospitals & 0.220 & 0.215 & 0.212 & - & - \\
\hline Internists $^{2)}$ & NA & 0.205 & 0.203 & 0.55 & 0.58 \\
\hline Surgeons ${ }^{3)}$ & NA & 0.234 & 0.236 & 0.87 & 0.87 \\
\hline Orthopedists & 0.193 & 0.182 & 0.179 & 0.62 & 0.63 \\
\hline Obstetricians/gynecologists & 0.224 & 0.228 & 0.235 & 0.56 & 0.59 \\
\hline Pediatricians & 0.217 & 0.211 & 0.215 & 0.59 & 0.61 \\
\hline Psychiatrists & 0.254 & 0.236 & 0.230 & 0.77 & 0.75 \\
\hline Brain surgeons & 0.262 & 0.239 & 0.231 & 0.84 & 0.85 \\
\hline Ophthalmologists & 0.246 & 0.222 & 0.221 & 0.37 & 0.36 \\
\hline Dermatologists & 0.279 & 0.263 & 0.261 & 0.41 & 0.41 \\
\hline Otolaryngologists & 0.253 & 0.233 & 0.230 & 0.40 & 0.41 \\
\hline Urologists & 0.277 & 0.246 & 0.233 & 0.73 & 0.73 \\
\hline Anesthesiologists & 0.377 & 0.344 & 0.333 & 0.93 & 0.94 \\
\hline Pathologists & NA & 0.440 & 0.405 & 0.98 & 0.98 \\
\hline Radiologists & 0.421 & 0.407 & 0.390 & 0.94 & 0.93 \\
\hline Emergency physicians & NA & 0.488 & 0.407 & 1.00 & 0.99 \\
\hline Rehabilitation physicians & 0.426 & 0.348 & 0.343 & 0.93 & 0.94 \\
\hline
\end{tabular}

NA, not available.

${ }^{1)}$ Gini coefficients were calculated for physicians working in hospitals or clinics. The number of physicians in each specialty was calculated as the sum of those in each SMA used in this study. We successfully extracted data for almost all the SMAs (342 out of 344).

${ }^{2}$ Internists included allergy physicians, cardiologists, diabetologists, gastroenterologists, hematologists, infectious disease internists, internal physicians, nephrologists, neurologists, psychotherapists, pulmonologists, and rheumatologists.

${ }^{3)}$ Surgeons included breast surgeons, cardiac surgeons, colon surgeons, cosmetic surgeons, gastric surgeons, general surgeons, plastic surgeons, respiratory surgeons, and tracheoesophageal surgeons.

4) We were unable to extract data for internists, surgeons, pathologists, and emergency physicians in 2000 because of data availability.

\section{Specialty-specific physician distribution}

Table 3 shows the Gini coefficient and the proportion of physicians working in hospitals for each specialty. The geographical distribution of overall physicians seemed to improve from 2000 through 2016 (the Gini coefficients were $0.227,0.220$, and 0.217 in 2000, 2010, and 2016, respectively), but the geographic distribution of physicians varied by specialty. In particular, hospital-based specialists, such as anesthesiologists, pathologists, radiologists, emergency physicians, and rehabilitation physicians, most of whom worked in hospitals, were more clustered. The Gini coefficients of most specialties were around 0.2 to 0.3 , but those of anesthesiologists, pathologists, radiologists, emergency physicians, and rehabilitation physicians were around 0.4. The distribution of $\mathrm{OB} / \mathrm{GYNs}$ seemed to get worse during the study period (from 0.224 in 2000 to 0.235 in 2016). This trend was in the opposite direction from other specialists.

\section{Physician distribution by hospital dependence}

The hospital-dependent specialties were anesthesiol- ogy, pathology, radiology, emergency medicine, and rehabilitation medicine. The other 11 specialties were considered non-hospital-dependent. Lorenz curves for each of these categories are shown in Fig. 1. These curves revealed that there was a distributional difference by hospital dependency. This tendency was preserved when the threshold of the hospital dependency was set at 0.8 (not shown). When the analysis was restricted to physicians working exclusively in hospitals (i.e., excluding any physicians who worked in clinics), hospital-dependent specialists were still more clustered than other specialists (Table 4).

\section{Specialty-specific physician distribution at prefecture level}

Table 5 shows the Gini coefficients of each specialty by prefecture. As a whole, the Gini coefficients were lower than in the SMA-level analyses, but the Gini coefficients of some specialties, such as psychiatry, dermatology, anesthesiology, pathology, radiology, emergency medicine, and rehabilitation medicine, were higher than other specialties. 


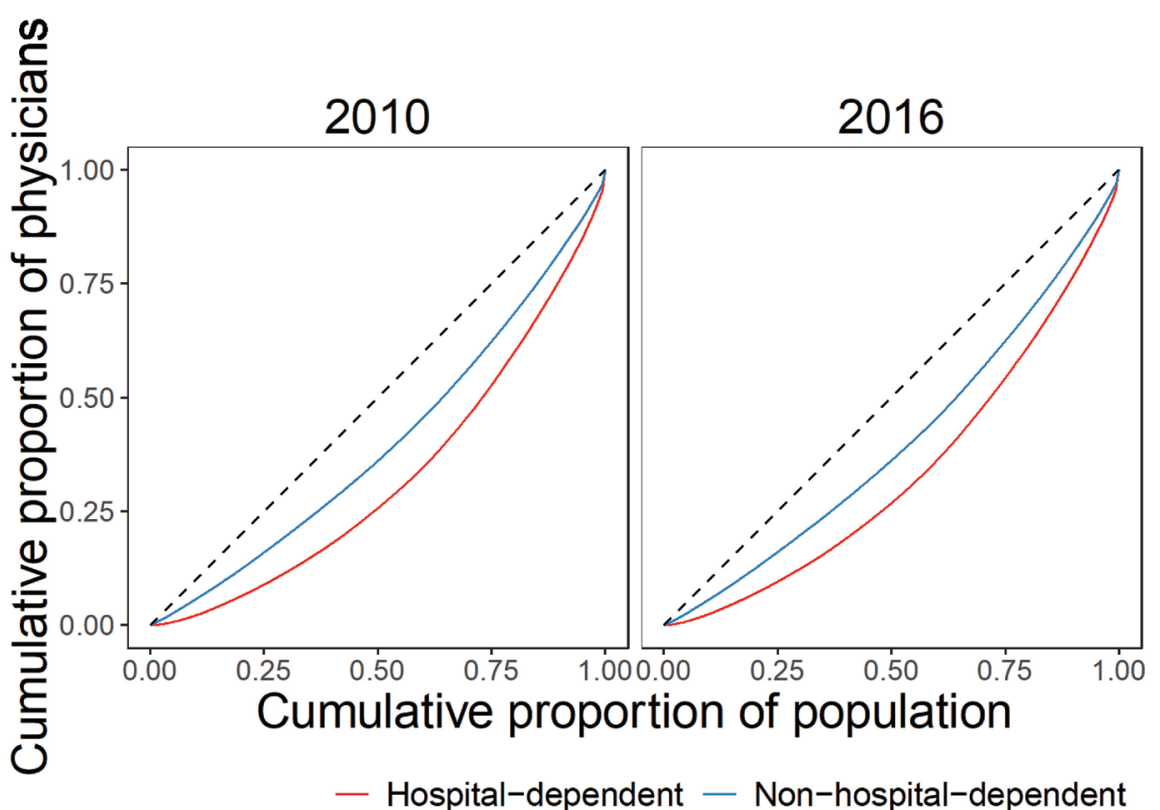

Fig. 1. Lorenz curves by specialty hospital dependency in 2010 and 2016.

Hospital-dependent specialties were defined as those in which at least $90 \%$ of specialists worked in hospitals.

The "hospital-dependent" group included anesthesiologists, pathologists, radiologists, emergency physicians, and rehabilitation physicians.

The "non-hospital-dependent" group included internists, surgeons, orthopedists, obstetricians/gynecologists, pediatricians, psychiatrists, brain surgeons, ophthalmologists, dermatologists, otolaryngologists, and urologists.

Table 4. Specialty-specific Gini coefficients for physicians working exclusively in hospitals ${ }^{1)}$.

\begin{tabular}{lll}
\hline & 2010 & 2016 \\
\hline Doctors working in hospitals & 0.264 & 0.256 \\
Internists $^{2}$ & 0.269 & 0.263 \\
Surgeons $^{3)}$ & 0.240 & 0.237 \\
Orthopedists & 0.233 & 0.224 \\
Obstetricians/gynecologists & 0.299 & 0.292 \\
Pediatricians & 0.295 & 0.294 \\
Psychiatrists & 0.266 & 0.257 \\
Brain surgeons & 0.256 & 0.253 \\
Ophthalmologists & 0.363 & 0.369 \\
Dermatologists & 0.389 & 0.396 \\
Otolaryngologists & 0.367 & 0.365 \\
Urologists & 0.279 & 0.270 \\
Anesthesiologists & 0.350 & 0.335 \\
Pathologists & 0.440 & 0.403 \\
Radiologists & 0.406 & 0.386 \\
Emergency physicians & 0.490 & 0.408 \\
Rehabilitation physicians & 0.369 & 0.356
\end{tabular}

1)The number of physicians in each specialty was calculated as the sum of the number in each SMA used in this study. We successfully extracted physician data for almost all the SMAs (342 out of 344).

2)Internists included allergy physicians, cardiologists, diabetologists, gastroenterologists, hematologists, infectious disease internists, internal physicians, nephrologists, neurologists, psychotherapists, pulmonologists, and rheumatologists.

${ }^{3}$ Surgeons included breast surgeons, cardiac surgeons, colon surgeons, cosmetic surgeons, gastric surgeons, general surgeons, plastic surgeons, respiratory surgeons, and tracheoesophageal surgeons. 
Table 5. Specialty-specific Gini coefficients in prefecture-level analyses ${ }^{1)}$.

\begin{tabular}{lccc}
\hline & $2000^{4)}$ & 2010 & 2016 \\
\hline All doctors & 0.121 & 0.116 & 0.112 \\
Doctors working in clinics or hospitals $^{2)}$ & 0.118 & 0.114 & 0.109 \\
Internists $^{2)}$ & $\mathrm{NA}$ & 0.122 & 0.118 \\
Surgeons $^{3}$ & $\mathrm{NA}$ & 0.121 & 0.118 \\
Orthopedists $^{\text {Obstetricians/gynecologists }}$ & 0.103 & 0.103 & 0.100 \\
Pediatricians & 0.103 & 0.100 & 0.107 \\
Psychiatrists & 0.098 & 0.100 & 0.102 \\
Brain surgeons & 0.136 & 0.134 & 0.129 \\
Ophthalmologists & 0.126 & 0.115 & 0.111 \\
Dermatologists & 0.139 & 0.123 & 0.119 \\
Otolaryngologists & 0.142 & 0.144 & 0.138 \\
Urologists & 0.137 & 0.125 & 0.118 \\
Anesthesiologists & 0.137 & 0.125 & 0.116 \\
Pathologists & 0.167 & 0.153 & 0.139 \\
Radiologists & $\mathrm{NA}$ & 0.169 & 0.155 \\
Emergency physicians & 0.221 & 0.205 & 0.192 \\
Rehabilitation physicians & NA & 0.242 & 0.181 \\
\hline NA, not avalable. & 0.232 & 0.178 & 0.177 \\
\hline
\end{tabular}

NA, not available.

1) These analyses included all 344 SMAs.

2)Internists included allergy physicians, cardiologists, diabetologists, gastroenterologists, hematologists, infectious disease internists, internal physicians, nephrologists, neurologists, psychotherapists, pulmonologists, and rheumatologists.

${ }^{3)}$ Surgeons included breast surgeons, cardiac surgeons, colon surgeons, cosmetic surgeons, gastric surgeons, general surgeons, plastic surgeons, respiratory surgeons, and tracheoesophageal surgeons.

${ }^{4)}$ We were unable to extract data for internists, surgeons, pathologists, and emergency physicians in 2000 because of data availability.

\section{Discussion}

The geographical distribution of overall physicians seemed to improve between 2000 and 2016 in terms of the Gini coefficients $(0.227,0.220$, and 0.217 in 2000, 2010, and 2016, respectively), but the geographic distribution of physicians in Japan varied by specialty. In particular, hospital-dependent specialties, where most physicians worked in hospitals (such as anesthesiologists, pathologists, radiologists, emergency physicians, and rehabilitation physicians), were more clustered, even after the numbers of such physicians particularly increased. The distribution of $\mathrm{OB} /$ GYNs worsened throughout the study period.

The improvement of the Gini coefficients of almost all of the specialists seems reasonable because the numbers of physicians increased during the study period. The underlying mechanism, however, may not be the increased number of physicians alone. As shown in a previous study, many rural areas suffered from decreasing populations over the study period (Tanihara et al. 2011). The Gini coefficient may therefore have been improved because the overall population, which is the denominator of the Gini coefficient, decreased. We inferred from population data that the smaller SMAs had experienced decreases in population
(Table 2). This reasoning is compatible with previous studies, which showed that the increasing number of physicians by itself did not ease physician maldistribution (Kobayashi and Takaki 1992; Toyokawa and Kobayashi 2010).

The increase of the Gini coefficient for OB/GYNs may be because of the integration of perinatal medical care systems in Japan. Since around 2005, the Japanese government has tried to integrate perinatal medical care systems instead of distributing physicians evenly (Ministry of Health, Labour and Welfare 2005). This is because of the limited number of physicians in charge of perinatal care and the resulting concerns for safety and burden of perinatal care. A previous study showed that the number of hospitals dealing with delivery in Japan declined from 1,321 in 2005 to 1,051 in 2011 (Matsumoto et al. 2015). Further research is needed to evaluate whether this integration has eased physicians' burdens without impairing access to perinatal care.

Hospital-based specialists, such as anesthesiologists, pathologists, radiologists, emergency physicians, and rehabilitation physicians, were more clustered than non-hospital-based specialists. This might be because of the distribution of hospitals. However, this tendency was observed even when we restricted our analyses to physicians working 
in hospitals. The uneven distribution among hospitaldependent specialists could therefore be at least partly a result of practice style. To further support this idea, we calculated Gini coefficients for hospital beds, using the Survey of Medical Institutions, which was open to the public (Ministry of Health, Labour and Welfare 2018). The Gini coefficients for hospital beds in SMAs were 0.170 and 0.168 in 2010 and 2016. These values were lower than the Gini coefficient for specialists (Table 3), which also supports our idea that the distribution of hospital-dependent specialists was not the result of the hospital distribution alone.

Hospital-dependent specialists may have unique aspects to their practice that could affect their geographical location. First, radiologists and anesthesiologists require expensive medical equipment such as radiographic image diagnosis apparatus, including computed tomography scanners, magnetic resonance imaging scanners, and positron emission tomography scanners, or anesthesia equipment. These physicians may therefore tend to co-locate to make better use of expensive medical equipment. Second, specialists such as pathologists, radiologists, and rehabilitation physicians could be distributed disproportionately so that they had enough work. Not all patients need diagnostic radiology examinations, pathological diagnosis, or rehabilitation. Our results may therefore reflect the fact that these specialists tend to work in bigger centers to secure sufficient work to keep their practice up-to-date. Finally, emergency medicine is a highly resource-intensive field. To scrutinize and treat patients comprehensively, emergency physicians are required to cooperate with other specialists and make use of medical equipment. For example, a patient with multiple trauma needs a computed tomography scan before appropriate treatments (and, in some cases, radiologists judge how badly the patient is injured). Furthermore, physicians from various specialties, such as surgery and orthopedic surgery, as well as emergency medicine, can be involved in the treatment.

Our results show that these hospital-dependent specialists were more clustered than other specialists, even at the prefecture level (Table 5). Overall, our results imply that the nature of some specialists' practice led to their disproportionate distribution, so that access to medical care in some fields is not consistent across the whole country.

Previous studies showed that overall physician distribution improved or remained stable over a similar period, so the result of this study is compatible with these results (Tanihara et al. 2011; Hara et al. 2018). Another previous study showed an association between specialists' distribution and their tendency to self-employment (Matsumoto et al. 2010). This is also compatible with our study, but our study is distinct from previous studies because we showed that the distribution of physicians in hospital-dependent specialties was consistently more clustered even when we restricted analyses to physicians working exclusively in hospitals. Our results therefore suggest that it is the nature of practice in the specialty that affects specialists' distribution, not the distribution of hospitals.

This study had some limitations. First, we did not adjust for the demand for care in each specialty, because there is no established way to do so. From the perspective of medical care delivery, it is essential to grasp the exact demand so that policymakers could balance the demand and supply of medical care. While demand for pediatricians and obstetricians may well decline as the number of children or pregnant women decreases in an area, demand for other specialties, such as internists, surgeons, and orthopedists, could increase as the population ages. However, demand for hospital-dependent specialties, such as anesthesiologists, pathologists, radiologists, and emergency physicians, are less likely to be related to population aging. Our results imply the nature of practice in some specialties may have led to a disproportional distribution and uneven supply of medical care in particular fields even after the numbers of physicians increased. This implication should be taken into consideration to implement effective measures for medical care delivery, as well as knowledge concerning demand and supply of medical care, which we hope will be provided through a further study. Second, the numbers of some specialists, such as pathologists, emergency physicians, and rehabilitation physicians, were so small that these specialists could not be distributed evenly using SMAs as the study unit. The population of the smallest SMA was only about 20,000 (Table 2). The total population used in this study was less than $127,000,000$, so about 6,350 (= $127,000,000 / 20,000)$ physicians would be needed for an even distribution. We therefore cannot tell whether these specialists were distributed evenly, because there were fewer than 5,000 in these specialties. The numbers of anesthesiologists, radiologists, and OB/GYNs were larger, however, so our discussion about the distribution of these specialists remains plausible. Finally, our results were based on data from Japan, and may not be applicable to other countries. Healthcare systems vary between countries, so the nature of each specialty may also vary. However, our study has identified the important point that the nature of practice varies by specialty, and this may affect the geographical distribution of specialists.

In conclusion, the geographical distribution of overall physicians in Japan appeared to have improved since 2000 in terms of the Gini coefficients, but the magnitude of the improvement varied by physicians' specialty. In particular, physicians in hospital-dependent specialties were more clustered even than their peers in other specialties. These results indicate that the nature of practice in each specialty strongly affected physician distribution. This suggests that it is important to pay attention to the nature of each specialty in discussing the distribution of physicians.

\section{Acknowledgments}

We would like to thank all the physicians who have contributed to the Physician, Dentist, and Pharmacist 
Census. We also thank Dr. Keiko Nakamura from Meikai University and Melissa Leffler MBA from Edanz Group (https://en-author-services.edanzgroup.com/) for reviewing and commenting on our manuscript.

This study was a part of the comprehensive research project headed by Prof. Soichi Koike, Jichi Medical University, and funded by the Japanese Ministry of Health, Labour and Welfare administration research grant [grant number: H30-Iryo-Shitei-014]. The funding agency had no role in the design and conduct of this study.

\section{Author Contributions}

All the authors contributed to the conception and design of this study, and drafting, reviewing, or revision of the article. R.I., A.M., and Y.K. designed the study. R.I. wrote the first draft, A.M. and Y.K. critically reviewed the manuscript, and R.I. revised the manuscript. All the authors approved the final version of the manuscript for submission.

\section{Conflict of Interest}

The authors declare no conflict of interest.

\section{References}

Chang, C.H., O’Malley, A.J. \& Goodman, D.C. (2017) Association between temporal changes in primary care workforce and patient outcomes. Health Serv. Res., 52, 634-655.

Chang, C.H., Stukel, T.A., Flood, A.B. \& Goodman, D.C. (2011) Primary care physician workforce and Medicare beneficiaries' health outcomes. JAMA, 305, 2096-2104.

Chang, R.K. \& Halfon, N. (1997) Geographic distribution of pediatricians in the United States: an analysis of the fifty states and Washington, DC. Pediatrics, 100, 172-179.

Dalton, H. (1920) The measurement of the inequality of incomes. Econ. J., 30, 348-361.

Goodfellow, A., Ulloa, J.G., Dowling, P.T., Talamantes, E., Chheda, S., Bone, C. \& Moreno, G. (2016) Predictors of primary care physician practice location in underserved urban or rural areas in the United States: a systematic literature review. Acad. Med., 91, 1313-1321.

Guttmann, A., Shipman, S.A., Lam, K., Goodman, D.C. \& Stukel, T.A. (2010) Primary care physician supply and children's health care use, access, and outcomes: findings from Canada. Pediatrics, 125, 1119-1126.

Hann, M. \& Gravelle, H. (2004) The maldistribution of general practitioners in England and Wales: 1974-2003. Br. J. Gen. Pract., 54, 894-898.

Hara, K., Kunisawa, S., Sasaki, N. \& Imanaka, Y. (2018) Examining changes in the equity of physician distribution in Japan: a specialty-specific longitudinal study. BMJ Open, 8, e018538.

Horev, T., Pesis-Katz, I. \& Mukamel, D.B. (2004) Trends in geographic disparities in allocation of health care resources in the US. Health Policy, 68, 223-232.

Huang, E.S. \& Finegold, K. (2013) Seven million Americans live in areas where demand for primary care may exceed supply by more than 10 percent. Health Aff. (Millwood), 32, 614-621.
Isabel, C. \& Paula, V. (2010) Geographic distribution of physicians in Portugal. Eur. J. Health Econ., 11, 383-393.

Kobayashi, Y. \& Takaki, H. (1992) Geographic distribution of physicians in Japan. Lancet, 340, 1391-1393.

MacDowell, M., Glasser, M., Fitts, M., Nielsen, K. \& Hunsaker, M. (2010) A national view of rural health workforce issues in the USA. Rural Remote Health, 10, 1531.

Matsumoto, M., Inoue, K., Bowman, R. \& Kajii, E. (2010) Selfemployment, specialty choice, and geographical distribution of physicians in Japan: a comparison with the United States. Health Policy, 96, 239-244.

Matsumoto, M., Koike, S., Matsubara, S., Kashima, S., Ide, H. \& Yasunaga, H. (2015) Selection and concentration of obstetric facilities in Japan: longitudinal study based on national census data. J. Obstet. Gynaecol. Res., 41, 919-925.

Ministry of Health, Labour and Welfare (2017) Medical care planning.

https://www.mhlw.go.jp/file/06-Seisakujouhou-10800000Iseikyoku/0000159901.pdf [Accessed: March 29, 2020](in Japanese).

Ministry of Health, Labour and Welfare (2005) Summary of the investigation committee on the intensification of pediatric and obstetric care.

https://www.mhlw.go.jp/shingi/2005/12/s1212-10g.html [Accessed: March 29, 2020](in Japanese).

Ministry of Health, Labour and Welfare (2018) Survey of medical institutions.

https://www.e-stat.go.jp/stat-search/files?page $=1 \&$ toukei $=004$ $50021 \&$ tstat $=000001030908$

[Accessed: March 29, 2020](in Japanese).

Ministry of Internal Affairs and Communications (2017) Basic resident registration network system.

https://www.e-stat.go.jp/stat-search/files?page=1\&layout=data list\&toukei $=00200241 \&$ tstat $=000001039591 \&$ cycle $=7 \&$ tc lass $1=000001039601$ [Accessed: March 29, 2020](in Japanese).

Newhouse, J.P. (1990) Geographic access to physician services. Annu. Rev. Public Health, 11, 207-230.

Petterson, S., Phillips, R.J., Bazemore, A. \& Koinis, G. (2013) Unequal distribution of the U.S. primary care workforce. https://www.aafp.org/afp/2013/0601/od1.html [Accessed: March 29, 2020].

Rosenthal, M.B., Zaslavsky, A. \& Newhouse, J.P. (2005) The geographic distribution of physicians revisited. Health Serv. Res., 40, 1931-1952.

Sakai, R., Fink, G., Kumamaru, H. \& Kawachi, I. (2016) The impact of pediatrician supply on child health outcomes: longitudinal evidence from Japan. Health Serv. Res., 51, 530-549.

Sharma, D.C. (2015) India still struggles with rural doctor shortages. Lancet, 386, 2381-2382.

Simoens, S. \& Hurst, J. (2006) The supply of physician services in OECD countries. http://www.oecd.org/els/health-systems/35987490.pdf [Accessed: March 29, 2020].

Tanihara, S., Kobayashi, Y., Une, H. \& Kawachi, I. (2011) Urbanization and physician maldistribution: a longitudinal study in Japan. BMC Health Serv. Res., 11, 260.

Toyokawa, S. \& Kobayashi, Y. (2010) Increasing supply of dentists induces their geographic diffusion in contrast with physicians in Japan. Soc. Sci. Med., 71, 2014-2019. 